\title{
Graph theory analysis of complex brain networks: new concepts in brain mapping applied to neurosurgery
}

\author{
Michael G. Hart, MBChB, ${ }^{1,2}$ Rolf J. F. Ypma, PhD, ${ }^{1,3}$ Rafael Romero-Garcia, PhD, ${ }^{1}$ \\ Stephen J. Price, FRCS (Neuro.Surg), ${ }^{2}$ and John Suckling, PhD ${ }^{1}$ \\ 1Brain Mapping Unit, Department of Psychiatry, and 2Division of Neurosurgery, Department of Clinical Neurosciences, \\ Addenbrooke's Hospital; and 'Hughes Hall, University of Cambridge, United Kingdom
}

\begin{abstract}
Neuroanatomy has entered a new era, culminating in the search for the connectome, otherwise known as the brain's wiring diagram. While this approach has led to landmark discoveries in neuroscience, potential neurosurgical applications and collaborations have been lagging. In this article, the authors describe the ideas and concepts behind the connectome and its analysis with graph theory. Following this they then describe how to form a connectome using resting state functional MRI data as an example. Next they highlight selected insights into healthy brain function that have been derived from connectome analysis and illustrate how studies into normal development, cognitive function, and the effects of synthetic lesioning can be relevant to neurosurgery. Finally, they provide a précis of early applications of the connectome and related techniques to traumatic brain injury, functional neurosurgery, and neurooncology.
\end{abstract}

http://thejns.org/doi/abs/10.3171/2015.4.JNS142683

KEY WORDS functional connectivity; functional neurosurgery; neurooncology; resting state networks; resting fMRI; traumatic brain injury

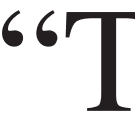
o make the delicate, awesome, and fateful work of the neurosurgeon more accurate, gentle and safe." This statement by Professor Albert L. Rhoton, one of the 20th century's most famous neurosurgeons and anatomists, encapsulates the objectives of both neurosurgical management and research. Achieving this goal for many fields of neurosurgery requires preserving or improving a patient's brain function. Thus, understanding functional neuroanatomy is fundamental to the advancement of surgical techniques and subsequent therapeutic strategies.

The human brain is the most complex system yet discovered, and understanding its form and function remains one of the greatest scientific challenges. The digital age has produced the necessary technologies and concepts to begin to make sense of the bewildering complexity of this most mysterious organ. Recently, ideas of the brain as a network of unceasing communication have emerged through comparison with other, common, natural and manmade systems that share key organizational principles. A combination of in vivo imaging, statistical modeling, and graph theoretical analysis has allowed the development of increasingly realistic models with explanatory and predic- tive properties. A paradigm shift has subsequently arisen, leading to the description of brain function as a consequence of information exchange between its components rather than information processing within individual components.

Here we aim to introduce complex brain networks and graph theory to the neurosurgical community. To begin, we explain how the brain can be viewed as a complex network and how graph theory can be used to explore the network's properties. With these ideas established we then describe how new avenues have been created in understanding functional brain anatomy, resilience, recovery, cognitive function, and disease biomarkers. Finally, we discuss how complex network analyses and graph theory have already been applied to "real-world" scenarios, including traumatic brain injury, neurooncology, and functional neurosurgery for psychiatric disease.

\section{Development of the Network Based Approach: a Historical Perspective}

Noninvasive, tomographic, in vivo functional neuroimaging began with the discovery of blood oxygenation level dependent (BOLD) endogenous contrast, ${ }^{72}$ where neuronal

ABBREVIATIONS BOLD = blood oxygenation level dependent; DBS = deep brain stimulation; DMN = default mode network; EEG = electroencephalography; fMRI = functional MRI; ICA = independent component analysis; $M E G$ = magnetoencephalography; $M R I=$ magnetic resonance imaging; $P E T$ = positron emission tomography; TBI = traumatic brain injury.

SUBMITTED December 7, 2014. ACCEPTED April 8, 2015.

INCLUDE WHEN CITING Published online November 6, 2015; DOI: 10.3171/2015.4.JNS142683. 
TABLE 1. Summary of neuroimaging techniques used to perform complex network analyses

\begin{tabular}{|c|c|c|c|c|}
\hline Technique & Acquisition & Application to Brain Networks & Strengths & Weaknesses \\
\hline Structural MRI & $\begin{array}{l}\text { Single 3D volume of the brain } \\
\text { (e.g., T1 MPRAGE), usually } \\
\text { acquired as standard in } \\
\text { most MRI protocols }\end{array}$ & $\begin{array}{l}\text { Analyses of structural covariance } \\
\text { of morphological measures } \\
\text { (e.g., cortical thickness or } \\
\text { volume) btwn brain regions } \\
\text { (high correlation implies a } \\
\text { network link) }\end{array}$ & $\begin{array}{l}\text { Simple to acquire \& not } \\
\text { limited by artifacts to } \\
\text { same degree as other } \\
\text { MRI-based sequences }\end{array}$ & $\begin{array}{l}\text { Limited by degree of inference } \\
\text { one can deduce based on } \\
\text { cortical measures }\end{array}$ \\
\hline Diffusion MRI & $\begin{array}{l}\text { Specific sequence using } \\
\text { gradients to measure free } \\
\text { water diffusion as a sur- } \\
\text { rogate measure of white } \\
\text { matter tracts }\end{array}$ & $\begin{array}{l}\text { Reconstruction of tracts can be } \\
\text { used to imply structural con- } \\
\text { nectivity btwn brain regions }\end{array}$ & $\begin{array}{l}\text { Suggests a clear correlation } \\
\text { w/ underlying structural } \\
\text { connectivity \& the brain's } \\
\text { "wiring diagram" }\end{array}$ & $\begin{array}{l}\text { Variations in sequences \& algo- } \\
\text { rithms can significantly affect } \\
\text { network parameters }\end{array}$ \\
\hline Functional MRI & $\begin{array}{l}\text { Specific 4D sequence } \\
\text { sensitive to BOLD contrast } \\
\text { reflecting hemodynamic re- } \\
\text { sponse of neuronal activity }\end{array}$ & $\begin{array}{l}\text { Analyses of statistical dependen- } \\
\text { cies btwn parcellated brain } \\
\text { regions; often performed in } \\
\text { "resting" state }\end{array}$ & $\begin{array}{l}\text { Reasonably high temporal \& } \\
\text { good spatial resolution }\end{array}$ & $\begin{array}{l}\text { Indirect measure of neuronal } \\
\text { activity; significant artifacts } \\
\text { require careful preprocessing }\end{array}$ \\
\hline EEG & $\begin{array}{l}\text { Uses electrodes to directly } \\
\text { measure electrical signals } \\
\text { in the brain }\end{array}$ & $\begin{array}{l}\text { Measures statistical dependen- } \\
\text { cy-for example, synchro- } \\
\text { nization likelihood-btwn } \\
\text { all pairwise combinations of } \\
\text { channels, often in multiple } \\
\text { frequency bands }\end{array}$ & $\begin{array}{l}\text { Best temporal resolution } \\
\text { \& direct measure of } \\
\text { (extracellular) neuronal } \\
\text { currents }\end{array}$ & $\begin{array}{l}\text { Significant spatial limitations \& } \\
\text { distortion due to skull \& scalp }\end{array}$ \\
\hline MEG & $\begin{array}{l}\text { Uses a magnetometer to } \\
\text { measures magnetic field } \\
\text { alterations due to neuronal } \\
\text { electrical activity }\end{array}$ & $\begin{array}{l}\text { Measures statistical dependency } \\
\text { btwn all pairwise combinations } \\
\text { of channels }\end{array}$ & $\begin{array}{l}\text { Exceptionally high temporal } \\
\text { resolution but limited spa- } \\
\text { tial resolution (particularly } \\
\text { subcortical) }\end{array}$ & $\begin{array}{l}\text { Difficulties w/ localizing signal } \\
\text { spatially \& accounting for } \\
\text { poor signal-to-noise ratio }\end{array}$ \\
\hline PET & $\begin{array}{l}\text { Injection of a radioisotope } \\
\text { followed by detection of } \\
\text { gamma rays }\end{array}$ & $\begin{array}{l}\text { Covariance in glucose metabo- } \\
\text { lism btwn regions }\end{array}$ & $\begin{array}{l}\text { Provides direct metabolic } \\
\text { data \& is a good biomark- } \\
\text { er for certain diseases } \\
\text { such as Alzheimer's } \\
\text { disease }\end{array}$ & $\begin{array}{l}\text { Radiation, limits on repeat- } \\
\text { ability, \& potentially lower } \\
\text { spatial resolution when not } \\
\text { combined w/ MRI }\end{array}$ \\
\hline
\end{tabular}

activity produces local blood oxygenation changes detectable by magnetic resonance imaging (MRI). The cognitive neuroscience community was an early adopter of this technology with a burgeoning in discoveries that certain brain functions are localized to anatomical regions, previously only surmised through lesioning studies or electrophysiological studies ${ }^{100}$ (with significant contributions from neurosurgery ${ }^{42}$ ). However, it was soon discovered, using positron emission tomography (PET), that task-induced activity accounts for only $1 \%-5 \%$ of the total energy budget of the brain, the most energetically expensive human organ. The great majority of brain metabolism is occurring continually, even in the absence of a cognitive stimulus ${ }^{75}$ when it is at "rest."

Changing the focus from task-based experiments to analyzing spontaneous activity while participants were resting revealed synchronous low-frequency $(<0.1 \mathrm{~Hz})$ fluctuations in the BOLD signal that formed coherent networks of neural activity. ${ }^{14}$ Central to this frame-shift in thinking was the discovery of the so-called default mode network (DMN), a synchronous set of dispersed brain regions continuously active at rest, but showing reduced activity during cognitively effortful tasks. ${ }^{38,39,95}$ The close relationship of the DMN, and other resting state networks, to task-based activation patterns ${ }^{84}$ implies an interconnect- edness between brain networks and the underlying structural connectivity. ${ }^{27,50}$

Describing the organizational characteristics and evolution of networks in both space and time has been the subject of many mathematical approaches (for example, dynamic causal modeling [DCM], ${ }^{12}$ independent component analysis [ICA $],{ }^{12}$ and graph theory $\left.{ }^{19}\right)$. While a network approach to brain function is not a new idea, ${ }^{25,49,68,85,88}$ a recent focus on brain connectivity as the underlying principle of the brain has gained eminence in the past decade, culminating in the search for the connectome, or the brain's "wiring diagram." 89 Synonymous with this rise in networks and connectomics has been the emergence of resting functional MRI (fMRI), although important contributions have come from a variety of neuroimaging modalities (Table 1) including electroencephalography (EEG), ${ }^{69}$ magnetoencephalography (MEG) $,{ }^{90} \mathrm{PET},{ }^{30,79}$ structural MRI, ${ }^{48}$ and diffusion based imaging. ${ }^{45,46}$ In short, there is now a large movement in neuroimaging studies toward viewing the functional integration or connectivity, superseding functional segregation and the localizationist viewpoint. ${ }^{41}$

\section{What Is a Complex Network?}

Graph theory was originally devised to solve real-world problems by viewing a system as an abstract network 


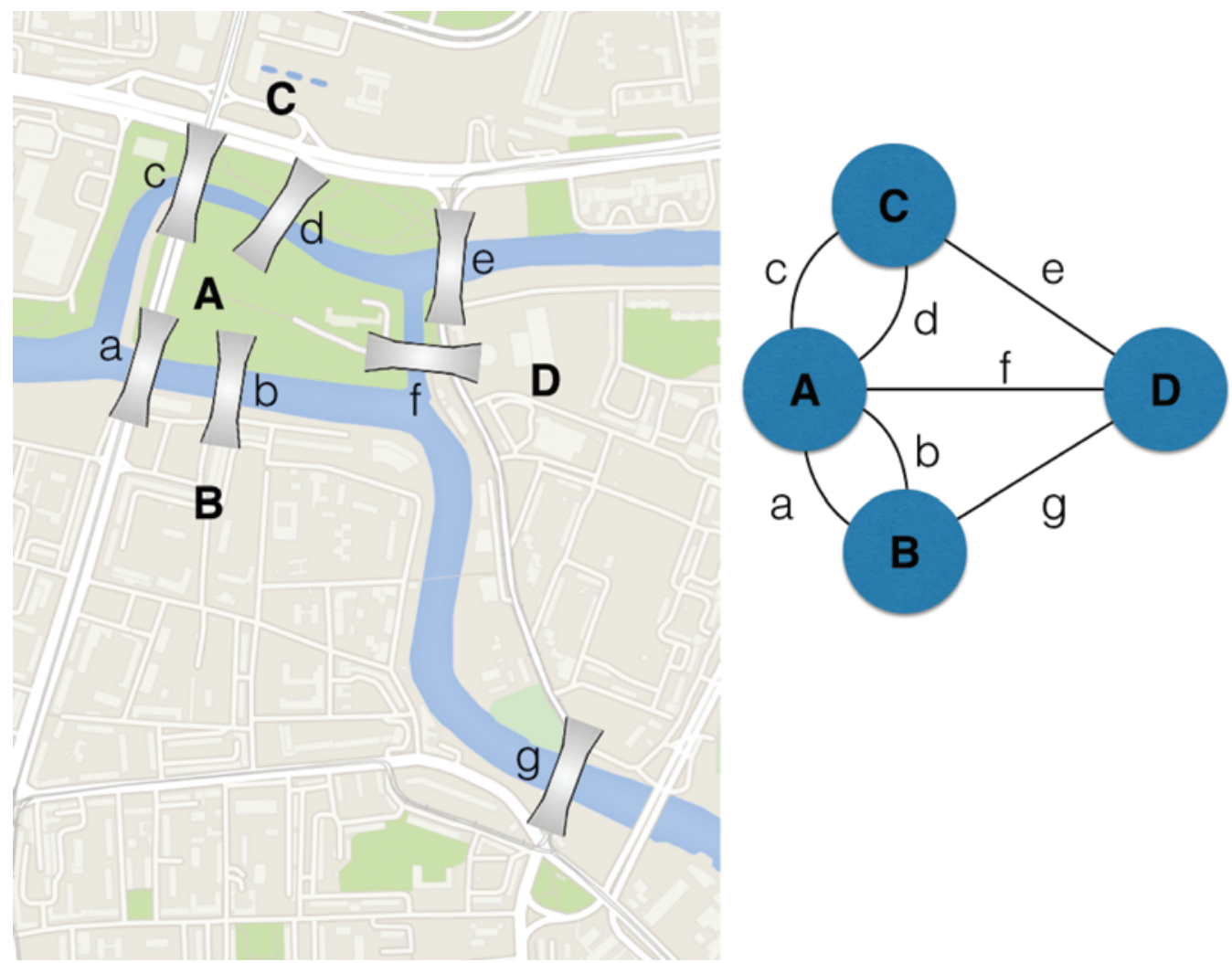

FIG. 1. The Königsberg bridge problem. The city of Königsberg in Prussia (now Kaliningrad in Russia) is set on the river Pregel and incorporates several islands connected by 7 bridges. The problem involved finding a way to walk through the city such that every bridge would be crossed once and only once. In 1735, Leonard Euler (1707-1783) mapped the problem out in terms of a simple graph, which allowed him to analyze the problem with mathematical rigor and generate a formal proof. It was shown that there was no solution, as for it to be true the graph needed less than 2 nodes of odd degree. The general term for traversing a network by passing each edge once and only once is now termed an "Eulerian walk." Figure is available in color online only.

(Fig. 1), which then allowed its properties to be analyzed mathematically. ${ }^{37}$ A network consists of point-like components, nodes or vertices, $\mathrm{V}$, and the relationships between them, links or edges, E. For example, when characterizing social relationships, nodes are individual people, and the presence or absence of an edge between 2 nodes indicates whether the corresponding people are friends or not. Together, the sets of nodes and edges form a binary "friendship" graph: $G=\{\mathrm{V}, \mathrm{E}\}$. Further information can be encoded; for example, the strength of the friendship can be represented by assigning a value, or weight, to the edges. Weighted or binary graphs can be directed, if an edge has an associated direction (for example, if each person were independently asked about their relationships with others in the network) or undirected, in which case no directionality in the relationships is implied.

The strength of graph theory is that once a graph has been constructed, the specific meanings of the nodes and edges becomes irrelevant, and the same analyses can therefore be applied to graphs originating from a wide spectrum of real-world networks.

\section{Network Measures}

Once the graph is formed, its properties (and therefore those of the real-life network it represents) can be captured mathematically (Fig. 2 and Table 2). In a binary graph the simplest property is the degree of a node, the number of connections or links that it has, which is a measure of how well the node is connected. The degree distribution is the histogram of node degrees for the overall network and is an important network property ${ }^{5}$ that can be used to distinguish between networks (described below). The total number of connections in a network can also be regarded as an estimate of the cost in establishing a particular configuration. ${ }^{1}$

Two network concepts of particular interest are segregation and integration. ${ }^{94}$ Network segregation relates to how well the network can be separated into constituent communities of nodes. A standard measure for determining segregation is clustering, which can be thought of as the tendency of nodes that share neighbors to be connected (e.g., are your friends each other's friends?).

Network integration measures the connectedness of distinct regions. One way of defining this is by the number of edges required to move from any one node to a target node, and the average of these values across all nodes in a network is known as the average path length. ${ }^{103}$ A related measure is global efficiency, which is the inverse of path length, ${ }^{60}$ but which has a greater relative contribution from short-distance connections. ${ }^{1}$ Note that these metrics are 


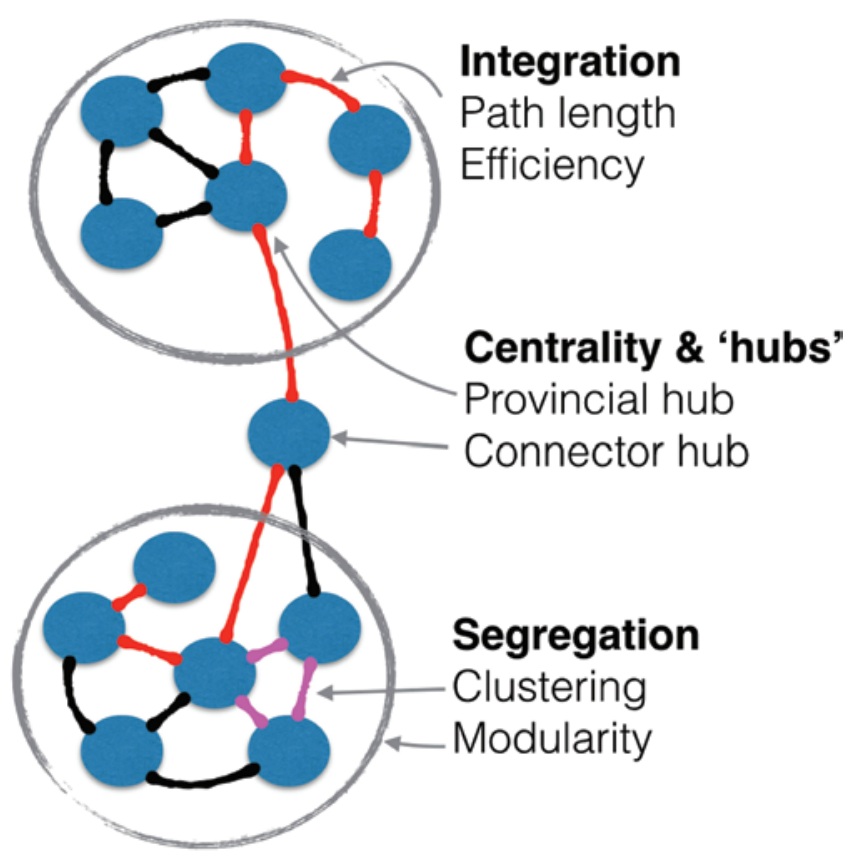

FIG. 2. Network measures. The main network measures and their classification are summarized in a hypothetical network illustration. Measures of segregation focus on community structure either in the form of small triangles (clustering) or larger groups of related components (modules). Measures of integration are based on the number of steps (edges) between individual nodes (known as the path length). The final main class of network measures are hubs, which can be defined in a variety of ways, depending either on how many links they have (degree), or how many paths. Figure is available in color online only.

restricted to topological connections and do not take into account geometrical distance between nodes.

\section{Network Models}

An alternative to computing a network's metrics, which are mainly descriptive, is to propose models of graph structure that explain the measured properties. ${ }^{3}$ Historically, 2 simple models, amenable to mathematical analysis, have been studied (Fig. 3). A lattice graph is a regular array of nodes with connections solely between adjacent nodes. This means that all connections are local, and therefore lattices show a high clustering coefficient but also a high path length (since getting from one edge of the graph to another requires traversing a large number of nodes). Another extreme is formed by the random graph, where nodes are connected by randomly placed links. This leads to few local connections, and therefore random graphs have low clustering, but short path lengths. Neither lattices nor random graphs represent accurate models of real-world networks.

Graph theory experienced a quantum leap in the late 1990s when 2 seminal papers showed that a range of real-world networks were well approximated by 2 elegant network models. The first of these created graphs by "rewiring" lattices-converting some of the short range connections into long-range connections (or "short cuts"). ${ }^{103}$ It was found that after only a few rewirings, the graphs showed a strongly reduced average path length, while maintaining high clustering. This property, where most interactions are local (high segregation) but where it is still possible to reach any part of the graph in a few steps (high integration), was dubbed the "small-world" property. It was found to be a ubiquitous property of seemingly disparate complex networks including the Internet, Hollywood actor collaborations, power grid organization, and neural networks.

Concurrent with the formulation of small-world networks, another feature found to be common in many realworld networks is the existence of a small number of highly connected nodes (called "hubs"). ${ }^{6}$ This network model could be generated using a simple process of sequentially adding nodes to a graph and preferentially attaching them to nodes that already had many connections (e.g., everyone wants to be friends with the popular person). The resulting degree distribution follows a power law. Many realworld networks have been found to exhibit this property, although often the distribution is capped due to physical restraints (e.g., the finite size of a brain), in which case an exponentially truncated power law degree distribution is appropriate. ${ }^{2}$

Since the 1990s, small-world networks and scale-free degree distributions have been the defining properties of complex networks with nontrivial topological features, contrasting with random graphs and lattices. Conservation of network properties over a wide range of fields has facilitated transdisciplinary sharing of rules for growth, evolution, and robustness between networks. The realization that these rules could model the behavior of real networks has led to the establishment and blossoming of network science over the past 2 decades and the application of complex networks to the central nervous systems of invertebrates and vertebrates, including humans.

\section{How Are Brain Networks Abstracted From Empirical Data?}

Methods of constructing graphs from diverse neuroimaging modalities share many similar concepts (Fig. 4), but with subtle differences that depend on the nature of the data. ${ }^{19,37}$ Multiple spatial scales of data can be accommodated, ranging from micro-scales (e.g., light microscopy and cytoarchitecture, typically $\sim 10 \mu \mathrm{m}$ ) through mesoscales (e.g., viral tracers, typically $\sim 0.1 \mathrm{~mm}$ ) to macroscales (e.g., MRI, typically $\sim 1 \mathrm{~mm}) .{ }^{86}$ Whatever data input and planned inferences are chosen subsequently define the consequent network as 1) structural, if anatomical data are used (e.g., diffusion imaging or cortical morphology); 2) functional, if temporally varying data are the basis (e.g., resting fMRI, EEG, MEG, or PET); or 3) effective, if the data are attempting to suggest causal influences between regions (i.e., a directed graph).

Recalling that both vertices and edges are required to construct a network, the first stage is to define the nodes. This may be straightforward-for example, the positions of EEG or MEG electrodes-or require division of tomographic brain imaging data by some arbitrary, although principled method, known as parcellation. ${ }^{107}$

Once nodes have been established, edges have to be defined. For data collected over time, such as EEG, MEG, 
TABLE 2. Glossary of network terminology

\begin{tabular}{|c|c|}
\hline Measure & Definition \\
\hline Adjacency matrix & A means of displaying the nodes of a network and the edges or relationship between each pair of nodes \\
\hline Centrality & $\begin{array}{l}\text { A measure that describes how critical a given nodes features are to the overall network; many varia- } \\
\text { tions of centrality are available reflecting whether they refer to the number of edges or paths that pass } \\
\text { through a region, for example }\end{array}$ \\
\hline Clustering & $\begin{array}{l}\text { A measure of segregation defined as the number of neighbors of a node that are also neighbors of each } \\
\text { other (and hence form a triangle); usually displayed as a coefficient based on the maximum possible } \\
\text { number of clusters }\end{array}$ \\
\hline Complex network & A network w/ non-trivial features, usually taken to refer to small-world or scale-free topology \\
\hline Connectome & $\begin{array}{l}\text { A term originally coined less than } 10 \text { years ago to describe the structural architecture of the brain that } \\
\text { guides the functional connections. }\end{array}$ \\
\hline Cost & $\begin{array}{l}\text { A measure of the connection density of a network; usually displayed as a percentage of the maximum } \\
\text { number of connections in a network; theoretically related to the wiring length cost involved in forming } \\
\text { connections btwn nodes }\end{array}$ \\
\hline Degree & The number of connections a node has w/ other nodes in the network \\
\hline Degree distribution & The histogram of the degrees of all nodes for the network \\
\hline Efficiency & $\begin{array}{l}\text { The inverse of path length; often more accurate than path length when used in sparsely connected } \\
\text { graphs such as the brain }\end{array}$ \\
\hline Hub & $\begin{array}{l}\text { A feature of a node that makes it pertinent to the network's overall characteristics; can be defined in a } \\
\text { variety of ways, such as on centrality or degree of the node }\end{array}$ \\
\hline Module & $\begin{array}{l}\text { A conglomeration of nodes that are more strongly connected to each other than nodes outside the } \\
\text { module, often defined using hierarchical clustering algorithms }\end{array}$ \\
\hline Parcellation & Partition of the brain into distinct regions or clusters \\
\hline Path length & $\begin{array}{l}\text { A measure of integration that describes the number of discrete steps between nodes (or edges) that are } \\
\text { required to move from one node to another }\end{array}$ \\
\hline Percolation theory & $\begin{array}{l}\text { A branch of mathematics that considers the connectivity of a graph \& is often used to model the effects } \\
\text { of disrupting the network in terms of how connected the majority of its components are }\end{array}$ \\
\hline Resting state & Refers to the brain's activity when not engaging in a task \\
\hline Rich club & $\begin{array}{l}\text { A group of nodes w/ highly central features (\& often key hubs) that are also highly connected to each } \\
\text { other (i.e., have high assortativity) }\end{array}$ \\
\hline Robustness & The ability of a network to sustain its characteristic features when either nodes or edges are removed \\
\hline
\end{tabular}

or resting fMRI, the strength of a connection between 2 nodes is frequently estimated by the Pearson correlation between their time series, although other methods can also be used. ${ }^{106}$ For data without temporal information such as estimates of brain structure from MRI (cortical thickness, gray matter volume, and cortical surface), edge strengths are the covariance of observations between individuals and thus represent the average connections within a group or population sample. ${ }^{48}$ Diffusion MRI uses a slightly different method whereby the number of tracts (calculated from a tractography algorithm) are calculated between parcels. $^{45,46}$

Once these calculations are complete the data are organized into a 2D adjacency matrix, where nodes are represented by rows and columns, and edge weights are indicated by the matrix entries, as each entry lies at the intersection of a row and column. After this point the methods of analysis are shared irrespective of the original data or the final objective of the analysis..$^{55}$ Once the graph has been created, its properties can be characterized using a range of graph theory measures. Toolboxes have been developed with optimized coding to provide efficient, reliable, and standardized computation of measures and methods for statistical testing. ${ }^{77}$

One of the most appealing aspects of networks is their representation or visualization that renders them in a manner which makes interpretation possible. ${ }^{66}$ However, currently there is no standardized way in which to do this, and the choice of technique depends on the data and planned inferences (Fig. 5). Traditionally, visualizing neuroimaging data, particularly that for task fMRI, has emphasized anatomical accuracy and clarity. However, for connectivity and graph theory analyses, the emphasis changes to the pattern of interactions between any 2 nodes.

\section{The Brain's Small World-New Insights From Graph Theory Into Anatomical Models}

A fundamental property of brain networks-either structural or functional-is that they demonstrate clear small-world characteristics; that is, they are simultaneously segregated (high clustering coefficient) and integrated (short path lengths). ${ }^{10,87,103}$ In this manner they parsimoniously balance the needs of localized specialization and global efficiency. This highly nonrandom pattern of 

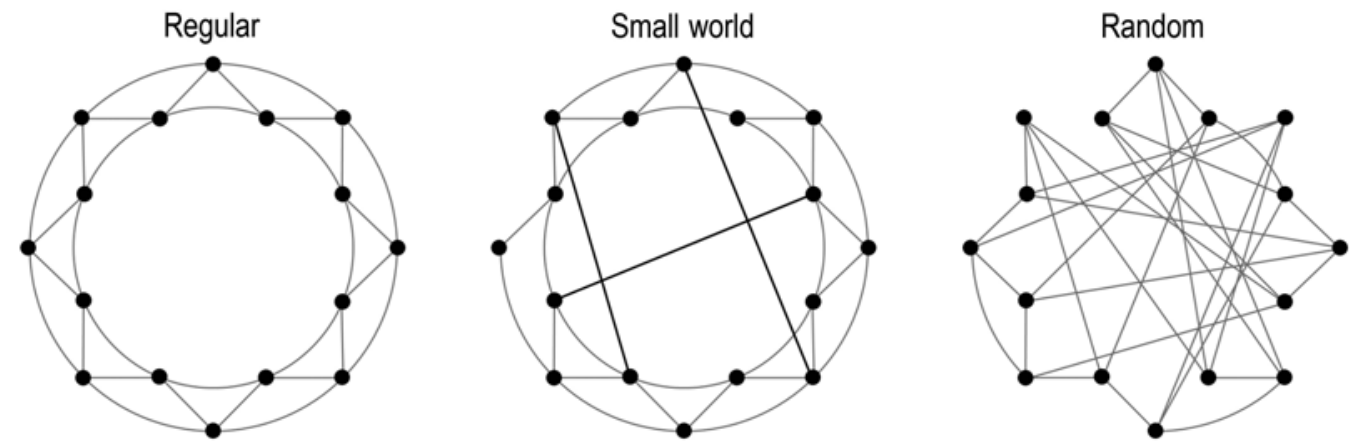

Increasing randomness

FIG. 3. Network types and the "small world." The 2 traditional classes of network models (lattice and random graphs) are shown on either end of a spectrum with small-world graphs lying in the middle. By rewiring a few of the short range connections in the lattice to any other node with a fixed probability, the small-world graph maintains high clustering (a key feature of the lattice) but dramatically reduces its path length (in keeping with a random graph), thereby parsimoniously balancing the features of segregation and integration. When the lattice has become completely rewired randomly, it has become a random graph.

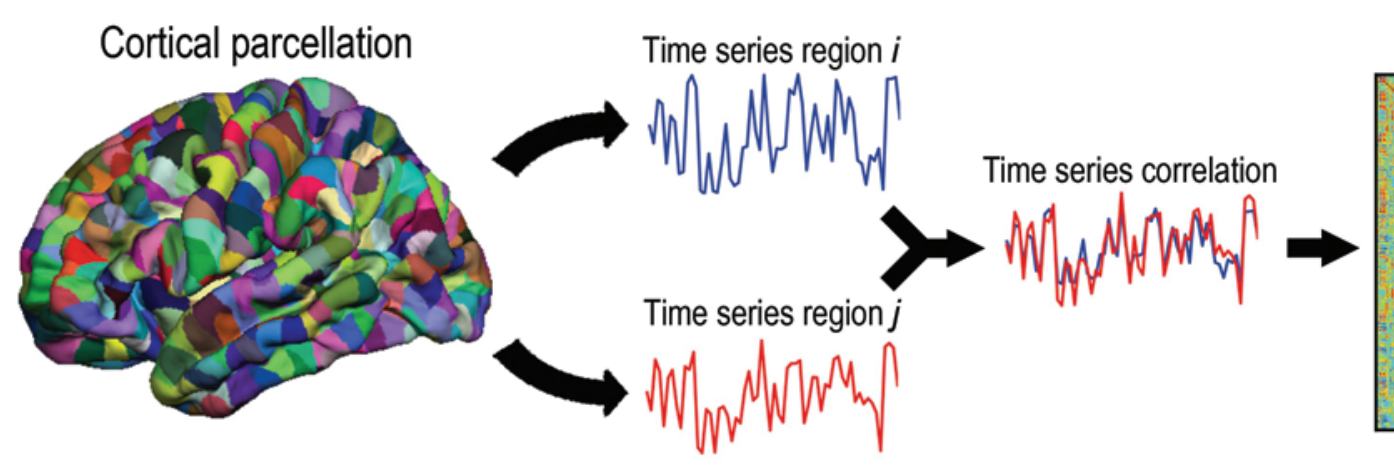

Connectivity matrix

Binarization and reordering nodes by modular structure
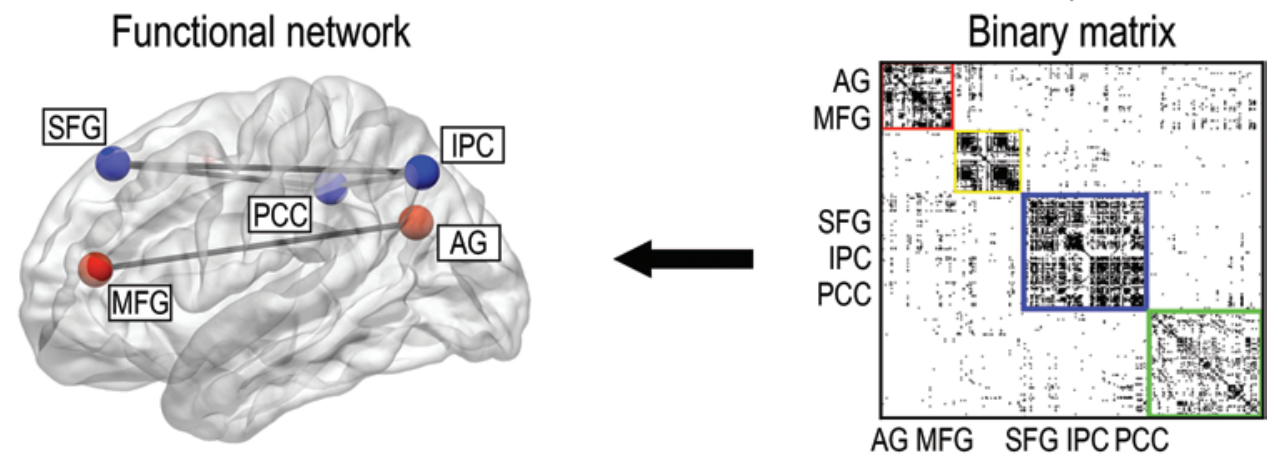

FIG. 4. Constructing a graph from resting fMRI data. First, the brain is parcellated into discrete regions (or nodes). In this case, a random parcellation is chosen based on an anatomical atlas but keeping the surface area constant. Subsequently, the mean time series of the 4D resting fMRI data are extracted for each parcel, and the relationship between each pair of parcels is calculated (known as an edge or a link). Usually this relationship is the Pearson correlation, although it can also be other measures, but it is essentially a form of statistical dependency. Once this has been done for each pair of parcels, the data are displayed in terms of an adjacency matrix that highlights the connectivity between the regions. In the matrix, rows and columns represent parcels (or nodes), and the relationships (or edges) are the entries in the matrix. The matrix can then be thresholded, binarized, or arranged in a hierarchical fashion. Finally, the connectivity data are translated back into anatomical space for display purpose. In this case it is overlaid with a translucent brain with lines to reflect significant links between regions, and the colors represent the module that the node belongs to. $A G=$ angular gyrus; $I P C=$ inferior parietal cortex; $M F G=$ middle frontal gyrus; $P C C=$ posterior cingulate cortex; $\mathrm{SFG}=$ superior frontal gyrus. Figure is available in color online only. 


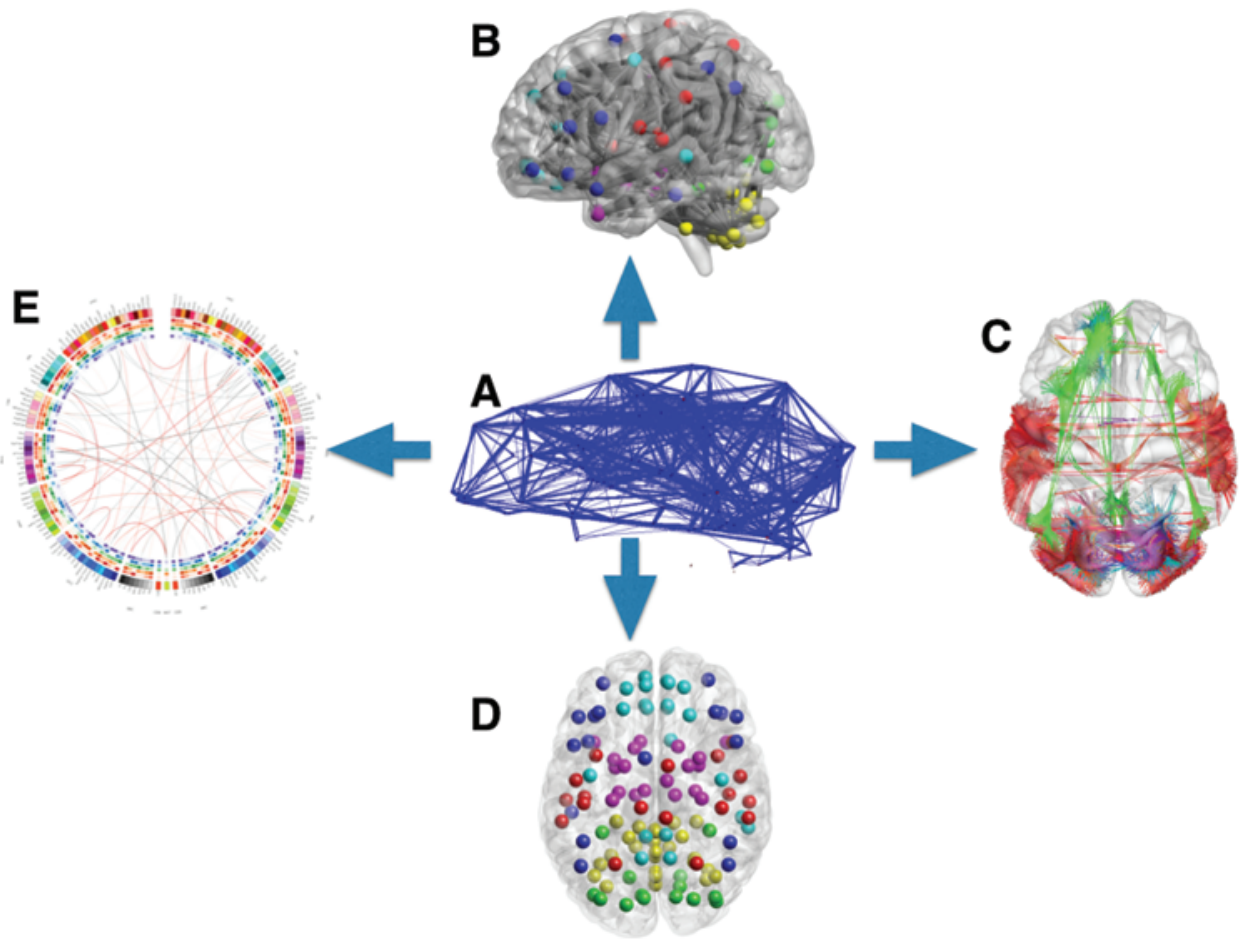

FIG. 5. Network visualization. In the simplest form, connectivity data can be represented in terms of a 2D adjacency matrix, although this does not take into account any spatial information. Translating connectivity patterns to physical space was originally performed in a $2 \mathrm{D}$ plane $(\mathrm{A})$, where lines were used to denote connections between regions in physical space. . $^{, 78} \mathrm{~A}$ weakness of this approach was that it often was not reconcilable with recognizable anatomy, a concern that can be allayed by rendering the wiring diagram on a surface reconstruction (B). A limitation of displaying heavily connected and detailed graphs is line clutter, whereby the graph appears to represent a lattice, and extracting information from the figure becomes impractical. Techniques borrowed from other complex network displays, such as hierarchical edge bundling, ${ }^{17}$ can be used to clarify the display of such information, although with the caveat that this can risk interpreting edges in brain networks as actual white matter tracts (C). If the function of individual nodes is of interest, varying node size or color can help illustrate their properties without requiring edges to be displayed (D). This can be of use when characterizing hubs and their associated "rich clubs," for example. Finally, more abstract renditions of connectivity data can be performed, whereby the display is detached almost completely from traditional anatomical localization, and instead emphasizes complexity and multivariate display of data (McGonigle et al: Visualising functional connectivity in fMRI using hierarchical edge bundles, poster presented at the 17th Annual Meeting of the Organization on Human Brain Mapping, Quebec City, Canada, 2011) ${ }^{52,53}$ (E). Movie representations have also been proposed to demonstrate dynamics in graph evolution. Figure is available in color online only.

connections is also remarkably sparse, in that the overall number of binary connections is a small proportion $(\sim$ $5 \%-10 \%)$ of the maximum number possible, emphasizing the highly organized nature of brain networks. ${ }^{105}$ Smallworld characteristics and sparse networks are complimentary features in that they demonstrate how simultaneous segregation and integration can be achieved at a low cost of connections. Sparse small-world attributes are robust in that they are reproducible across imaging modalities (diffusion MRI, ${ }^{45,46}$ resting fMRI, $, 278,102$ structural MRI, ${ }^{48}$ EEG, ${ }^{82}$ MEG, ${ }^{90}$ and PET $^{30,79}$ ), disease states, and species (such as the cat, macaque, and nematode Caenorhabditis elegans)..$^{20}$

The degree distribution of brain networks is difficult to accurately reproduce due to the small number of overall connections using common parcellations. ${ }^{56}$ However, it appears that brain networks do not demonstrate scale-free properties in their degree distribution, but rather have an exponentially truncated power law. ${ }^{2}$ This is not entirely unexpected given the space constraints involved. In other words, it would be difficult to imagine having sufficient room for a brain growing inside a closed space like the skull to accommodate superconnected regions that would reside in the extreme tail of the degree distribution. ${ }^{5}$ Other topologically constrained networks such as transportation networks and internet routers face similar issues. ${ }^{6}$

Highly connected nodes have been identified through a variety of means ${ }^{7,40}$ that quantify how central these nodes are in the network. ${ }^{96}$ These hubs are believed to play a key role in facilitating the flow of information in the network, but it is unlikely that they are eloquent per se. Furthermore, the distribution of connections between such hubs is often found to be significantly nonrandom, with highly connected hubs even more strongly connected to each other in a so-called "rich club." 97

Community detection is performed by grouping nodes into communities or modules such that they are more strongly connected to nodes within their own module than to those in other modules. ${ }^{71}$ Modules derived from a graph theoretical treatment are consistent with those identified 
using other means of assessing brain connectivity. ${ }^{95}$ These modules can be further broken down into submodules in a hierarchical manner. ${ }^{78}$ Within these modules, highly connected hubs have been identified which are further subclassified as either connector hubs if they link modules, or provincial hubs if they mainly integrate nodes within a module. ${ }^{44,88}$ Modularity reflects local specialization, but it also allows cost-effective network integration by adding a few long-range connections between modules.

Rules for neural network growth have been developed that try to accurately predict the behavior of real networks. For instance, when new nodes are connected preferentially to a highly connected "rich club," the degree distribution matches that of other real-world networks, a scenario known as "the rich get richer." Other models include the aging of vertices, whereby a certain number of nodes disappear over time, a process that replicates scale-free degree distributions. ${ }^{5}$ Clarifying the constraints and stimuli for network growth may allow insights into brain repair or adaptation after injury.

It should now be apparent that network models of brain structure and function aim to describe how the simultaneous demands of functional segregation and integration are met. In this new perspective, the focus changes from a localization approach, in which particular cognitive functions takes place in specified brain regions, to an integrative or connectomics based approach that emphasizes information flow across the entire network. Additionally, the vocabulary that accompanies graph theory offers a new way of expressing the exploration of the brain. A prominent structural and functional core can be defined based on specific hubs, modules, and efficiency, which can be used to re-explore classical models of brain activity. Whether differences in network parameters can be identified with enough reliability to encompass individual variability, dynamic reconfiguration, and evolutionary changes remains to be seen.

\section{Applications of Graph Theory in Neurosurgery: a New Concept in Brain Mapping}

While graph theory analysis of complex networks has allowed significant advances in our understanding of normal brain structure and function, in order to be clinically relevant it must also make neurosurgery "more accurate, gentle, and safe." We now discuss how graph theory can be applied to brain mapping, and in doing so give a new perspective that sees function from a connectivity-based perspective, rather than as purely localized. Supplementing traditional methods of brain mapping (such as intraoperative cortical stimulation), graph theory also allows prediction of dynamic changes (possibly in a reparative manner), as well as predicting how the brain can adapt (or not) to the presence of focal lesions, including those purposefully induced by surgery.

\section{Understanding Function}

Graph theory has characterized brain functional organization as a highly efficient, small-world network. Under- standing how this network architecture affects intellectual function may enable the abstract concepts and measures of graph theory to develop clinically relevant meaning. For example, certain network features may reflect the capacity of an individual to perform a specific neurocognitive task.

To understand the relationship between function and individual networks, comparison with statistical techniques such as ICA have been particularly insightful. Networks identified through ICA of fMRI data in the absence of an external stimulus were compared spatially to those derived during experiments that required cognitive engagement. ${ }^{84}$ A close correspondence was found between behavioral domains in the corresponding task-based fMRI and resting fMRI networks, suggesting that at baseline the brain is already organized along functional boundaries. It can then be hypothesized that such modules (and hierarchical submodules) represent a repertoire of functional networks that can be called upon for task-directed activities.

Experiments examining intelligence and network topology found that a higher IQ was negatively correlated with path length but not with clustering or overall connectivity. ${ }^{98}$ As a longer path length is inversely proportional to efficiency, this suggests that network efficiency is a key factor for cognitive function. Furthermore, the medial prefrontal cortex and precuneus (among other regions) were identified as having the greatest effect on network organization and global network efficiency. Notably, this study is a demonstration of how task performance patterns are reflected in functional connectivity at rest.

This critical role of path length and its inverse, efficiency, has been corroborated in a study examining brain structural networks. ${ }^{63}$ High intelligence corresponds to higher global efficiency in both weighted and binary graphs. During the performance of a task, a more prominent small-world architecture was found in those without higher education than in those with a university education, suggesting that more efficient network communication was required in the former group to complete the task. ${ }^{69}$ Thus, efficiency appears to be the key determinant of intellectual function. Relating network topology to specific neuropsychological tasks and to the effects of cortical stimulation findings will be critical to translating graph theoretical measures to clinically meaningful phenotypes.

\section{Characterizing Plasticity}

Brain networks are not static but are dynamic over a wide range of time scales: from seconds to years. Normal development offers an ideal opportunity to measure the long-term evolution of their properties. In terms of neurosurgical relevance, characteristic patterns of network dynamics could be used as a proxy for plasticity and to explain recovery from focal neurological disorders.

Development of a single specific brain network, the default mode network (DMN), has been studied in a cohort of children aged 7-9 years in comparison with adults aged 21-31 years..$^{34,35}$ In children, the DMN was sparsely connected and tended to follow anatomical (or local) patterns in its connections, whereas in adults, the DMN had developed into a more densely connected network encompassing the characteristic DMN regions. An earlier analysis of 
the same data found that developmental connectivity was characterized by an increase in long-range connections and decrease in short-range connections with increasing age, suggesting that as the graphs became less sparse, connections also changed from being anatomically to functionally coupled. ${ }^{35}$

Refinement of connections and the role of path length was investigated in a whole-brain network model using combined structural and functional imaging data. ${ }^{93}$ With increasing age there was a reduction of short-range connections and an increase in long-range connectivity. This is analogous to processes occurring at cellular scales where overconnectivity is refined through selective pruning. By adulthood, networks had also developed an increased hierarchy, and the interregional connectivity patterns had changed to form stronger cortico-cortical, but weaker subcortical-cortical connections.

Characteristic patterns of development have allowed machine-learning algorithms to predict brain maturation patterns with $92 \%$ accuracy. ${ }^{31}$ Weakening of short-range functional connections between major brain functional modules was the most important factor in the model, confirming the importance of connection pruning to overall development. Other identified changes over time included strengthening of long-range connections and increased segregation and modularity. Overall this produced modules that were more segregated from each other, but more densely connected within themselves.

These findings suggest that networks demonstrate characteristic patterns of dynamics over the 2 or more decades of brain development, converging to a more cohesive, efficient, and modular topology. ${ }^{29,62}$ Network models of development appear complimentary to traditional models, for example Hebbian learning, whereby repeated stimulation of one cell by another leads to increased synaptic efficiency. ${ }^{101}$ Determining the rules behind these network dynamics will require understanding of how they relate to neurocognitive traits, learning, and evolution.

From a neurosurgical perspective, lesions (either pathological or surgically induced) could trigger mechanisms that normally occur during development to repair the network. Using these principles, a description of how a brain network reconnects following attenuation or removal of specific nodes and edges could lead to an explanation of adaptation and reconstituted function. Potentially, rehabilitation could be tailored to the expected dynamic cascade of effects, or lack thereof.

\section{Modeling the Effects of Lesions}

Analysis of the effects of lesions on brain function has a long tradition of providing insights into functional localization, ${ }^{76}$ which has been complimented by high-resolution neuroimaging. ${ }^{11}$ However, understanding brain function from a localization perspective fails to account for dynamic changes, individual variability, and neurocognitive processes that require distributed rather than discrete processing. Network-based analysis of lesion effects offers a complementary consideration of system-wide functional disturbance. In addition, models can be readily created to virtually examine the effects of postulated lesions.
Network models can be computationally "lesioned" by either randomly removing nodes ("random error") or targeting removal of certain nodes based on their specific parameters, e.g., removing highly central hubs ("targeted attack"). The robustness of the network can be calculated by comparing the topology after lesioning with the prior, intact network. An exponentially truncated power law degree distribution has been found to confer robustness to random error but also leaves the network vulnerable to specific removal of hubs. ${ }^{2}$ Rather than brain development selecting for such robustness, it is more likely that it is a byproduct of the formation of developing efficient processing (for example, through a rich-club organizational topology). ${ }^{56}$

Modeling the effect of larger, potentially more realistic lesions was performed on a structural connectivity data set with simulated neural dynamics. ${ }^{4}$ Structural networks were robust to random node deletion or targeted removal of nodes based on their degree or strength, but vulnerable to targeted removal of highly central "hub" nodes. Simulated dynamic effects of lesions varied in size and spatial pattern depending on the lesion location, with lesions in the midline as well as those involving the temporoparietal junction and superior frontal gyrus tending to have the greatest effect on neural dynamics. In general, lesions reduced functional connectivity, with effects most pronounced in the ipsilateral hemisphere, but also extending in a nonlocal manner to the contralateral hemisphere. Finally, the extent that alterations of the structural network produced dynamic consequences was most accurately predicted by injury to the DMN rather than, for instance, the degree or strength of connections directly incorporated by the lesion. How these simulated lesions and corresponding network disruption relate to actual neuropsychological findings in patients is awaiting confirmation. However, it is likely that the relationship between simulated and in vivo lesions is considerably more complex and includes dynamic reparative mechanisms.

White matter or structural disconnectivity and its effects on function networks have been studied in a model of structural networks created from empirical data followed by a computer simulation of resting-state BOLD signals using the network as a substrate. Randomly removing links or decreasing the global coupling strength resulted in characteristic patterns of increased hierarchy, efficiency, and robustness, but reduced small-worldness and clustering and generated a narrower degree distribution. ${ }^{21}$ This pattern of altered network dynamics was found to be similar to that found in patients with schizophrenia, suggesting that altered structural connectivity could be responsible for dynamic and phenotypic changes. However, the effects of focal lesions are unlikely to be explained purely in terms of alterations to structural connectivity, and will most likely require a model combining structural and functional factors.

A network approach has revealed key features in terms of the brain's robustness and resilience to lesioning. Application of theory from other complex systems in response, for example, to the rerouting of traffic after road closures $^{26,28}$ or percolation theory and the degree to which networks can be impaired ("slowed down") before critical function is affected (as opposed to directly removing 
a node $)^{23}$ offer potential for understanding the subtleties of changes in information flow due to lesions. Network models of lesion effects could also be elaborated to encompass dynamic changes and potential neurocognitive functional consequences. Cognitive deficits could then be predicted based on the vulnerability of a network to attack and its potential for repair.

More speculatively, accurate lesion modeling could be used in intraoperative brain mapping to define the extent of resection or the effects of a surgical intervention. One can envision classifying brain regions into those that are highly vulnerable, subserve critical function, or have limited potential for recovery, and thus need to be preserved. On the other hand, regions may be highly resilient, have limited functional importance, or have significant dynamic potential for reorganization. In this case a safer resection could be predicted with little functional consequence.

\section{Review of Current and Potential Applications of Graph Theory in Neurosurgery Traumatic Brain Injury}

Traumatic brain injury (TBI) is a heterogeneous condition encompassing a wide range of pathologies and potential outcomes. Network-based approaches offer potential to increase our understanding of disease pathophysiology, mechanisms underlying neurocognitive deficits, and the progress and effects of rehabilitation.

Defining patterns of injury based on functional connectivity could provide biomarkers to aid in differential diagnosis and prognosis with greater validity than can be obtained from radiological assessment of standard clinical MRI sequences. In a study of patients with mild TBI, networks had a longer average path length, reduced overall cost, and reduced network efficiency compared with controls. ${ }^{73}$ In addition, TBI characteristically produced injury focused on the posterior cingulate cortex, known to be a critical hub in the normally functioning brain. Increasing the validity of these measures may allow individual prediction of a given injury's effect on the network as well as identification of characteristic disease "fingerprints" corresponding to specific pathologies.

Assessing TBI in terms of network dysfunction has allowed reappraisal of pathologies that have previously proven enigmatic. For example, blast-related head injuries are a relatively recent phenomenon characterized by minimal abnormalities on standard structural MRI. ${ }^{47}$ However, network measures are grossly deranged, with higher modularity and lower average participation coefficient in patients with these injuries. Thus, certain injuries could be defined in terms of their functional connectivity.

Subtle cognitive deficits that involve significant functional impairment are now being appreciated clinically even after minor head injury and concussion. Mechanisms of cognitive deficits after head injury have been investigated using graph theory measures. In a study of adults with minor TBI, the function of a given network was compared with that of healthy controls in a task-switching cognitive challenge. ${ }^{22}$ Task performance was significantly poorer in patients than in controls. Patients had increased connectivity and local efficiency compared with controls, both of which were correlated with task proficiency and severity of TBI. It is proposed that these connectivity markers reflect network reorganization in an attempt to compensate for the injury.

Rehabilitation after TBI is now being given increased clinical priority, but exactly how to tailor and assess the effectiveness of such therapy is contentious. Reorganization of functional networks has been found to occur during neuro-rehabilitation. Baseline measurements soon after TBI reproduced patterns of network disruption identified in previous studies (including increased path length and increased connectivity); during recovery, these measures returned to normal levels. ${ }^{24}$ Recovery of network measures also correlated positively with neurocognitive recovery, enhancing their validity and providing clinical meaning. This opens up the possibility that network measures could be a sensitive and objective means of monitoring recovery.

Finally, a connectomics approach has been used to cast new light on the notable example of head injury suffered by Phineas Gage. ${ }^{99}$ A simulated trajectory of the culprit iron bar was overlaid on a structural connectome from a healthy volunteer to model the extent of injury to gray and white matter. While this indeed confirmed notable local injury, it also identified patterns of widespread and longrange connectivity loss that could also have contributed to the characteristic postinjury behavioral patterns described.

Potential future avenues for network analysis and graph theory in TBI are extensive. ${ }^{81}$ Injuries that do not have significant associated structural changes (i.e., without focal injury such as hematomas or contusions) are ideal for graph theory analysis, as they simplify preprocessing of the data and comparison with example networks. Models of lesion effects that encompass brain function, plasticity, and resilience could be adapted to guide rehabilitation programs. Biomarkers based on network measures could have multiple applications in clinical trials, for example in selecting homogenous trial populations based on graph theory characteristics or in objective means of assessing the effects of cognitiion-enhancing medications.

\section{Deep Brain Stimulation for Neuropsychiatric Conditions}

A disconnectivity hypothesis has been proposed to explain certain neurological and psychiatric diseases. $.^{19,25} \mathrm{Ab}-$ errant connectivity of a structural or functional network (for example in terms of impaired network integration or segregation) is proposed to lead to the development of clinical symptoms. Deep brain stimulation (DBS) has been used for psychiatric conditions, but its mechanism of action is poorly understood, and the effects are capricious. ${ }^{58}$

Defining psychiatric diseases partly based on network parameters allows the effectiveness of DBS to be assessed in terms of how it is able to reset functional connectivity to within the normal range. In patients with obsessivecompulsive disorder, DBS of the nucleus accumbens normalized frontostriatal connectivity from excessive levels. ${ }^{34}$ A positive correlation between symptomatic relief and reduction in excess frontostriatal connectivity was found, implying that restoring network activity is an important objective for effective treatment.

Other psychiatric diseases that have been modeled with graph theory and are of potential relevance for DBS 
include depression and posttraumatic stress disorder. In depression, connectivity within the default mode network and fronto-thalamo-caudate regions was reduced, ${ }^{57}$ community structure was rearranged, ${ }^{65}$ and hubs were altered. ${ }^{64}$ Furthermore, depressed patients and healthy controls could be automatically classified with multivariate pattern analysis (94\% accuracy) ${ }^{108}$ and support vector machines (99\% accuracy). ${ }^{65}$ If these network configurations prove to be robust biomarkers, they could be used to stratify patients into homogeneous groups in clinical trials or indeed as selection criteria in themselves.

Network-based outcome assessment has also been applied in a study of DBS of the periventricular/periaqueductal gray (PVG/PAG) for chronic phantom limb pain. ${ }^{59}$ During stimulation, subjective symptom relief was correlated with significantly increased activity in the left midanterior orbitofrontal cortex and right subgenual cingulate cortex, both of which are known to be involved in pain relief. Moreover, when the stimulator was turned off, worsening symptoms were associated with reduced activity in the same areas, yielding a robust and reproducible effect of stimulation.

Combining functional connectivity analysis and the effects of DBS can also improve our understanding of disease pathophysiology, particularly when there is a lack of realistic animal models, such as for chronic pain. DBS of the anterior cingulate cortex in chronic pain produced stimulation-specific relief of symptoms that was accompanied by diffuse functional connectivity changes in the pre-supplementary motor area, brainstem periaqueductal gray matter, rostral anterior cingulate, and medial prefrontal areas..$^{70}$ As well as depicting the widespread networks involved in chronic pain, this study also improves understanding of the effects of stimulating the anterior cingulate cortex, which can be an effective DBS target for a range of conditions, including depression and obsessivecompulsive disorder.

Further studies combining DBS and complex network analyses may allow individual tailoring of therapy based on features of the abnormal network. For example, the specific manner in which a network is perturbed in depression may vary between individuals, and this could be used to identify an individualized target for stimulation. Refinement of graph theory biomarkers could potentially aid titration of stimulation parameters that optimize network reconfiguration.

\section{Neurooncology}

A fundamental goal in the resection of intrinsic brain lesions is to maximize the extent of resection for oncological gain ${ }^{91,92}$ while preserving brain function ${ }^{67}$ and ensuring a good quality of life. Current surgical methods in neurooncology, including awake surgery, ${ }^{80}$ cortical mapping, ${ }^{28}$ and neurophysiological techniques, ${ }^{13}$ have led to significant advancements in patient outcome and the emergence of supramarginal resections whereby tumor resections are extended to functional boundaries. ${ }^{54}$ Brain mapping has played a key role in the field since the pioneering days of Penfield ${ }^{74}$ and identification of the sensorimotor homunculus, while more recent studies have suggested that a hierarchical topology is involved in restoring function after lesions. ${ }^{33}$ Network science and graph theory offer a new language with which to further our understanding of functional organization and plasticity in response to brain tumors.

An abnormal signature of brain connectivity has been identified in patients with brain tumors. ${ }^{8,9,104}$ In general, brain networks in patients with tumors have been found to be more random and less organized, not just locally but in a diffuse manner. Specific findings include reduced efficiency in patients with tumors in the frontal and temporal but not parietal lobes. Also, network hubs were reorganized, with the right insula being a hub in controls but not in patients. A focused analysis including exclusively participants with frontal lobe tumor confirmed reduced local efficiency but increased global efficiency, as well as reduced clustering. ${ }^{51}$ Whether these global network dynamics reflect compensatory mechanisms or possible nonlocal effects of the tumor is awaiting clarification.

For graph theory measures to have clinical relevance it is necessary to relate network disruption to the patient's symptoms and function. The degree to which network features are perturbed has been correlated with neuropsychological deficits involving both local and diffuse brain regions with reduced global efficiency and small-worldness correlated with lower IQ scores. ${ }^{15,16,104}$ Applying graph theory measures to identify the neuropsychological function of a network allows a novel means to map out a functional resection boundary depending on the anticipated cognitive consequences.

Surgical effects on networks and how this corresponds to clinical outcomes have been measured. ${ }^{43,51}$ Networks have been found to be restored to a more organized state after surgery from a more disorganized preoperative state. Additionally, a specific pattern of preoperative network disruption was able to predict neurocognitive outcome. ${ }^{43}$ Regions that showed decreased coherence could be resected without new deficits appearing, while increased connectivity was associated with eloquent tissue. Seizure outcome has also been correlated with improvement in network characteristics. ${ }^{32}$ In this case, there was a trend for patients without seizures to have a larger decrease in interhemispheric connectivity in the theta band. Modeling the effects of surgery and the predicted network effects may thus offer a new avenue in preoperative surgical planning. For example, surgery could be tailored to produce a predicted network effect that prevents new neuropsychological deficits or optimizes seizure outcome.

These preliminary studies demonstrate the potential of using network measures to assess clinical function and the effects of surgery in patients with brain tumors. Modeling the effects of lesions (and surgery) on networks offers a way to analyze the predicted effects of surgery on a network, which in turn could be used to tailor the extent of resection based on the anticipated functional consequences and potential for network reorganization. This final concept will involve unification of concepts of brain function, plasticity (re-connectomics), and network vulnerability.

\section{Conclusions}

Complex network analysis and graph theory offer new horizons for exploring the effects of focal and diffuse pa- 
thologies managed in neurosurgery. Although the field is relatively new, its transdisciplinary nature means it has developed on validated concepts from associated areas, such as the social sciences and communication engineering. Already our understanding of neuropsychiatric conditions has advanced through applying these techniques, but so far applications within neurosurgery have been relatively rare. Specific roles for network science in neurosurgery include mapping brain function, characterizing plasticity, and modeling the brain's response to injury.

Ultimately though, the challenge will be to apply this understanding to patients to enhance clinical improvement of their management. ${ }^{18,61}$ Part of the translational efficacy will lie with improved technical standards in creating graphs to make them more reliable and intuitive. ${ }^{83}$ For instance, developing fully connected weighted graphs without arbitrary thresholding or developing realistic null models to benchmark network characteristics. Once this is in place, applying a network analysis to standard clinical MRI scans could feasibly become routine. Nevertheless, the most attractive opportunity is probably in integrating realistic models of brain lesions (factoring in function, plasticity, and robustness) to preoperative planning and neuronavigation as integral to surgical planning. Basic science research into functional brain mapping has aligned itself along a path that considers the brain as a product of its connections rather than isolated functional locations: integrating these advancements into clinical brain mapping will therefore be at the forefront of making neurosurgery "more accurate, gentle, and safe."

\section{Acknowledgments}

Dr. Hart is funded by the Wellcome Trust Neuroscience in Psychiatry Network with additional support from the National Institute for Health Research Cambridge Biomedical Research Centre.

\section{References}

1. Achard S, Bullmore E: Efficiency and cost of economical brain functional networks. PLOS Comput Biol 3:e17, 2007

2. Achard S, Salvador R, Whitcher B, Suckling J, Bullmore E: A resilient, low-frequency, small-world human brain functional network with highly connected association cortical hubs. J Neurosci 26:63-72, 2006

3. Albert R, Barabasi AL: Statistical mechanics of complex networks. Rev Mod Phys 74:47, 2002

4. Alstott J, Breakspear M, Hagmann P, Cammoun L, Sporns O: Modeling the impact of lesions in the human brain. PLOS Comput Biol 5:e1000408, 2009

5. Amaral LAN, Scala A, Barthelemy M, Stanley HE: Classes of small-world networks. Proc Natl Acad Sci U S A 97:11149-11152, 2000

6. Barabási AL, Albert R: Emergence of scaling in random networks. Science 286:509-512, 1999

7. Barthelemy M: Betweenness centrality in large complex networks. Eur Phys J B 38:163-168, 2004

8. Bartolomei F, Bosma I, Klein M, Baayen JC, Reijneveld JC, Postma TJ, et al: Disturbed functional connectivity in brain tumour patients: evaluation by graph analysis of synchronization matrices. Clin Neurophysiol 117:20392049, 2006

9. Bartolomei F, Bosma I, Klein M, Baayen JC, Reijneveld JC, Postma TJ, et al: How do brain tumors alter functional con- nectivity? A magnetoencephalography study. Ann Neurol 59:128-138, 2006

10. Bassett DS, Bullmore E: Small-world brain networks. Neuroscientist 12:512-523, 2006

11. Bates E, Wilson SM, Saygin AP, Dick F, Sereno MI, Knight RT, et al: Voxel-based lesion-symptom mapping. Nat Neurosci 6:448-450, 2003

12. Beckmann CF, DeLuca M, Devlin JT, Smith SM: Investigations into resting-state connectivity using independent component analysis. Philos Trans R Soc Lond B Biol Sci 360: 1001-1013, 2005

13. Bello L, Gallucci M, Fava M, Carrabba G, Giussani C, Acerbi F, et al: Intraoperative subcortical language tract mapping guides surgical removal of gliomas involving speech areas. Neurosurgery 60:67-82, 2007

14. Biswal B, Yetkin FZ, Haughton VM, Hyde JS: Functional connectivity in the motor cortex of resting human brain using echo-planar MRI. Magn Reson Med 34:537-541, 1995

15. Bosma I, Douw L, Bartolomei F, Heimans JJ, van Dijk BW, Postma TJ, et al: Synchronized brain activity and neurocognitive function in patients with low-grade glioma: a magnetoencephalography study. Neuro Oncol 10:734-744, 2008

16. Bosma I, Reijneveld JC, Klein M, Douw L, van Dijk BW, Heimans JJ, et al: Disturbed functional brain networks and neurocognitive function in low-grade glioma patients: a graph theoretical analysis of resting-state MEG. Nonlinear Biomed Phys 3:9, 2009

17. Böttger J, Schäfer A, Lohmann G, Villringer A, Margulies DS: Three-dimensional mean-shift edge bundling for the visualization of functional connectivity in the brain. IEEE Trans Vis Comput Graph 20:471-480, 2014

18. Bullmore E: The future of functional MRI in clinical medicine. Neuroimage 62:1267-1271, 2012

19. Bullmore E, Sporns O: Complex brain networks: graph theoretical analysis of structural and functional systems. Nat Rev Neurosci 10: 186-198, 2009

20. Bullmore E, Sporns O: The economy of brain network organization. Nat Rev Neurosci 13:336-349, 2012

21. Cabral J, Hugues E, Kringelbach ML, Deco G: Modeling the outcome of structural disconnection on resting-state functional connectivity. Neuroimage 62:1342-1353, 2012

22. Caeyenberghs K, Leemans A, Heitger MH, Leunissen I, Dhollander T, Sunaert S, et al: Graph analysis of functional brain networks for cognitive control of action in traumatic brain injury. Brain 135:1293-1307, 2012

23. Callaway DS, Newman ME, Strogatz SH, Watts DJ: Network robustness and fragility: percolation on random graphs. Phys Rev Lett 85:5468-5471, 2000

24. Castellanos NP, Leyva I, Buldú JM, Bajo R, Paúl N, Cuesta P, et al: Principles of recovery from traumatic brain injury: reorganization of functional networks. Neuroimage 55:1189-1199, 2011

25. Catani M, ffytche DH: The rises and falls of disconnection syndromes. Brain 128:2224-2239, 2005

26. Csányi G, Kertész J: Scaling behaviour in discrete traffic models. J Phys Math Gen 28:L427-L432, 1995

27. Damoiseaux JS, Greicius MD: Greater than the sum of its parts: a review of studies combining structural connectivity and resting-state functional connectivity. Brain Struct Funct 213:525-533, 2009

28. De Benedictis A, Moritz-Gasser S, Duffau H: Awake mapping optimizes the extent of resection for low-grade gliomas in eloquent areas. Neurosurgery 66:1074-1084, 2010

29. Dennis EL, Thompson PM: Mapping connectivity in the developing brain. Int J Dev Neurosci 31:525-542, 2013

30. Di X, Biswal BB: Metabolic brain covariant networks as revealed by FDG-PET with reference to resting-state fMRI networks. Brain Connect 2:275-283, 2012 
31. Dosenbach NU, Nardos B, Cohen AL, Fair DA, Power JD, Church JA, et al: Prediction of individual brain maturity using fMRI. Science 329:1358-1361, 2010

32. Douw L, Baayen H, Bosma I, Klein M, Vandertop P, Heimans J, et al: Treatment-related changes in functional connectivity in brain tumor patients: a magnetoencephalography study. Exp Neurol 212:285-290, 2008

33. Duffau H: A new concept of diffuse (low-grade) glioma surgery. Adv Tech Stand Neurosurg 38:3-27, 2012

34. Fair DA, Cohen AL, Dosenbach N: The maturing architecture of the brain's default network. Proc Natl Acad Sci U S A 105:4028-4032, 2008

35. Fair DA, Dosenbach NUF, Church JA, Cohen AL, Brahmbhatt S, Miezin FM, et al: Development of distinct control networks through segregation and integration. Proc Natl Acad Sci U S A 104:13507-13512, 2007

36. Figee M, Luigjes J, Smolders R, Valencia-Alfonso CE, van Wingen G, de Kwaasteniet B, et al: Deep brain stimulation restores frontostriatal network activity in obsessive-compulsive disorder. Nat Neurosci 16:386-387, 2013

37. Fornito A, Zalesky A, Breakspear M: Graph analysis of the human connectome: promise, progress, and pitfalls. Neuroimage 80:426-444, 2013

38. Fox MD, Buckner RL, Liu H, Chakravarty MM, Lozano AM, Pascual-Leone A: Resting-state networks link invasive and noninvasive brain stimulation across diverse psychiatric and neurological diseases. Proc Natl Acad Sci U S A 111:E4367-E4375, 2014

39. Fox MD, Raichle ME: Spontaneous fluctuations in brain activity observed with functional magnetic resonance imaging. Nat Rev Neurosci 8:700-711, 2007

40. Freeman LC: Centrality in social networks conceptual clarification. Soc Networks 1:215-239, 1978

41. Friston KJ: Functional and effective connectivity: a review. Brain Connect 1:13-36, 2011

42. Greenblatt SH, Dagi TF, Epstein MH: A History of Neurosurgery: In its Scientific and Professional Contexts. Park Ridge, IL: AANS, 1997

43. Guggisberg AG, Honma SM, Findlay AM, Dalal SS, Kirsch $\mathrm{HE}$, Berger MS, et al: Mapping functional connectivity in patients with brain lesions. Ann Neurol 63:193-203, 2008

44. Guimera R, Amaral LISAN: Functional cartography of complex metabolic networks. Nature 433:895-900, 2005

45. Hagmann P, Cammoun L, Gigandet X, Meuli R, Honey CJ, Wedeen VJ, et al: Mapping the structural core of human cerebral cortex. PLoS Biol 6:e159, 2008

46. Hagmann P, Kurant M, Gigandet X, Thiran P, Wedeen VJ, Meuli R, et al: Mapping human whole-brain structural networks with diffusion MRI. PLoS One 2:e597, 2007

47. Han K, Mac Donald CL, Johnson AM, Barnes Y, Wierzechowski L, Zonies D, et al: Disrupted modular organization of resting-state cortical functional connectivity in U.S. military personnel following concussive 'mild' blastrelated traumatic brain injury. Neuroimage 84:76-96, 2014

48. He Y, Chen ZJ, Evans AC: Small-world anatomical networks in the human brain revealed by cortical thickness from MRI. Cereb Cortex 17:2407-2419, 2007

49. Hebb DO: The effect of early and late brain injury upon test scores, and the nature of normal adult intelligence. Proc Am Philosoph Soc 85:275-292, 1942

50. Honey CJ, Thivierge J-P, Sporns O: Can structure predict function in the human brain? Neuroimage 52:766-776, 2010

51. Huang Q, Zhang R, Hu X, Ding S, Qian J, Lei T, et al: Disturbed small-world networks and neurocognitive function in frontal lobe low-grade glioma patients. PLoS One 9:e94095, 2014

52. Irimia A, Chambers MC, Torgerson CM, Filippou M, Hovda DA, Alger JR, et al: Patient-tailored connectomics visualization for the assessment of white matter atrophy in traumatic brain injury. Front Nuerol 3:10, 2012

53. Irimia A, Chambers MC, Torgerson CM, Van Horn JD: Circular representation of human cortical networks for subject and population-level connectomic visualization. Neuroimage 60:1340-1351, 2012

54. Ius T, Angelini E, Thiebaut de Schotten M, Mandonnet E, Duffau H: Evidence for potentials and limitations of brain plasticity using an atlas of functional resectability of WHO grade II gliomas: towards a "minimal common brain." Neuroimage 56:992-1000, 2011

55. Kaiser M: A tutorial in connectome analysis: topological and spatial features of brain networks. Neuroimage 57:892-907, 2011

56. Kaiser M, Martin R, Andras P, Young MP: Simulation of robustness against lesions of cortical networks. Eur J Neurosci 25:3185-3192, 2007

57. Korgaonkar MS, Fornito A, Williams LM, Grieve SM: Abnormal structural networks characterize major depressive disorder: a connectome analysis. Biol Psychiatry 76:567574, 2014

58. Kringelbach ML, Green AL, Aziz TZ: Balancing the brain: resting state networks and deep brain stimulation. Front Integr Neurosci 5:8, 2011

59. Kringelbach ML, Jenkinson N, Green AL, Owen SLF, Hansen PC, Cornelissen PL, et al: Deep brain stimulation for chronic pain investigated with magnetoencephalography. Neuroreport 18:223-228, 2007

60. Latora V, Marchiori M: Efficient behavior of small-world networks. Phys Rev Lett 87:198701, 2001

61. Lee MH, Smyser CD, Shimony JS: Resting-state fMRI: a review of methods and clinical applications. AJNR Am J Neuroradiol 34:1866-1872, 2013

62. Lenroot RK, Giedd JN: Brain development in children and adolescents: insights from anatomical magnetic resonance imaging. Neurosci Biobehav Rev 30:718-729, 2006

63. Li Y, Liu Y, Li J, Qin W, Li K, Yu C, et al: Brain anatomical network and intelligence. PLOS Comput Biol 5:e1000395, 2009

64. Lim HK, Jung WS, Aizenstein HJ: Aberrant topographical organization in gray matter structural network in late life depression: a graph theoretical analysis. Int Psychogeriatr 25:1929-1940, 2013

65. Lord A, Horn D, Breakspear M, Walter M: Changes in community structure of resting state functional connectivity in unipolar depression. PLoS One 7:e41282, 2012

66. Margulies DS, Böttger J, Watanabe A, Gorgolewski KJ: Visualizing the human connectome. Neuroimage 80:445461, 2013

67. McGirt MJ, Mukherjee D, Chaichana KL, Than KD, Weingart JD, Quinones-Hinojosa A: Association of surgically acquired motor and language deficits on overall survival after resection of glioblastoma multiforme. Neurosurgery 65:463-470, 2009

68. Mesulam MM: Large-scale neurocognitive networks and distributed processing for attention, language, and memory. Ann Neurol 28:597-613, 1990

69. Micheloyannis S, Pachou E, Stam CJ, Vourkas M, Erimaki $\mathrm{S}$, Tsirka V: Using graph theoretical analysis of multi channel EEG to evaluate the neural efficiency hypothesis. Neurosci Lett 402:273-277, 2006

70. Mohseni HR, Kringelbach ML, Probert Smith P, Green AL, Parsons CE, Young KS, et al: Application of a nullbeamformer to source localisation in MEG data of deep brain stimulation. Conf Proc IEEE Eng Med Biol Soc 2010:4120-4123, 2010

71. Newman ME: Fast algorithm for detecting community structure in networks. Phys Rev E Stat Nonlin Soft Matter Phys 69:066133, 2004 
72. Ogawa S, Tank DW, Menon R, Ellermann JM, Kim SG, Merkle H, et al: Intrinsic signal changes accompanying sensory stimulation: functional brain mapping with magnetic resonance imaging. Proc Natl Acad Sci U S A 89:59515955, 1992

73. Pandit AS, Expert P, Lambiotte R, Bonnelle V, Leech R, Turkheimer FE, et al: Traumatic brain injury impairs smallworld topology. Neurology 80:1826-1833, 2013

74. Penfield W, Rasmussen T: The Cerebral Cortex of Man. New York: Macmillan, 1950

75. Raichle ME, Mintun MA: Brain work and brain imaging. Annu Rev Neurosci 29:449-476, 2006

76. Rorden C, Karnath HO: Using human brain lesions to infer function: a relic from a past era in the fMRI age? Nat Rev Neurosci 5:813-819, 2004

77. Rubinov M, Sporns O: Complex network measures of brain connectivity: uses and interpretations. Neuroimage 52:1059-1069, 2010

78. Salvador R, Suckling J, Schwarzbauer C, Bullmore E: Undirected graphs of frequency-dependent functional connectivity in whole brain networks. Philos Trans R Soc Lond B Biol Sci 360:937-946, 2005

79. Sanabria-Diaz G, Martínez-Montes E, Melie-Garcia L: Glucose metabolism during resting state reveals abnormal brain networks organization in the Alzheimer's disease and mild cognitive impairment. PLoS One 8:e68860, 2013

80. Sanai N, Mirzadeh Z, Berger MS: Functional outcome after language mapping for glioma resection. N Engl J Med 358:18-27, 2008

81. Sharp DJ, Scott G, Leech R: Network dysfunction after traumatic brain injury. Nat Rev Neurol 10:156-166, 2014

82. Stam CJ, Jones BF, Nolte G, Breakspear M, Scheltens P: Small-world networks and functional connectivity in Alzheimer's disease. Cereb Cortex 17:92-99, 2007

83. Smith SM: The future of FMRI connectivity. Neuroimage 62:1257-1266, 2012

84. Smith SM, Fox PT, Miller KL, Glahn DC, Fox PM, Mackay $\mathrm{CE}$, et al: Correspondence of the brain's functional architecture during activation and rest. Proc Natl Acad Sci U S A 106: $13040-13045,2009$

85. Sporns O: Discovering the Human Connectome. Cambridge, MA: MIT Press, 2012

86. Sporns O: Networks of the Brain, ed 1. Cambridge, MA: MIT Press, 2010

87. Sporns O, Chialvo DR, Kaiser M, Hilgetag CC: Organization, development and function of complex brain networks. Trends Cogn Sci 8:418-425, 2004

88. Sporns O, Honey CJ, Kötter R: Identification and classification of hubs in brain networks. PLoS One 2:e1049, 2007

89. Sporns O, Tononi G, Kötter R: The human connectome: A structural description of the human brain. PLOS Comput Biol 1:e42, 2005

90. Stam CJ: Functional connectivity patterns of human magnetoencephalographic recordings: a 'small-world' network? Neurosci Lett 355:25-28, 2004

91. Stummer W, Meinel T, Ewelt C, Martus P, Jakobs O, Felsberg J, et al: Prospective cohort study of radiotherapy with concomitant and adjuvant temozolomide chemotherapy for glioblastoma patients with no or minimal residual enhancing tumor load after surgery. J Neurooncol 108:8997, 2012

92. Stummer W, Pichlmeier U, Meinel T, Wiestler OD, Zanella F, Reulen HJ: Fluorescence-guided surgery with 5-aminolevulinic acid for resection of malignant glioma: a randomised controlled multicentre phase III trial. Lancet Oncol 7:392-401, 2006

93. Supekar K, Musen M, Menon V: Development of large- scale functional brain networks in children. PLoS Biol 7:e1000157, 2009

94. Tononi G, Sporns O, Edelman GM: A measure for brain complexity: relating functional segregation and integration in the nervous system. Proc Natl Acad Sci U S A 91:50335037, 1994

95. van den Heuvel MP, Hulshoff Pol HE: Exploring the brain network: a review on resting-state fMRI functional connectivity. Eur Neuropsychopharmacol 20:519-534, 2010

96. van den Heuvel MP, Sporns O: Network hubs in the human brain. Trends Cogn Sci 17:683-696, 2013

97. van den Heuvel MP, Sporns O: Rich-club organization of the human connectome. J Neurosci 31:15775-15786, 2011

98. van den Heuvel MP, Stam CJ, Kahn RS, Hulshoff Pol HE: Efficiency of functional brain networks and intellectual performance. J Neurosci 29:7619-7624, 2009

99. Van Horn JD, Irimia A, Torgerson CM, Chambers MC, Kikinis R, Toga AW: Mapping connectivity damage in the case of Phineas Gage. PLoS One 7:e37454, 2012

100. Varela F, Lachaux JP, Rodriguez E, Martinerie J: The brainweb: phase synchronization and large-scale integration. Nat Rev Neurosci 2:229-239, 2001

101. Vértes PE, Alexander-Bloch A, Bullmore ET: Generative models of rich clubs in Hebbian neuronal networks and large-scale human brain networks. Philos Trans R Soc Lond B Biol Sci 369:20130531, 2014

102. Wang J, Zuo X, He Y: Graph-based network analysis of resting-state functional MRI. Front Syst Neurosci 4:16, 2010

103. Watts DJ, Strogatz SH: Collective dynamics of "smallworld" networks. Nature 393:440-442, 1998

104. Xu H, Ding S, Hu X, Yang K, Xiao C, Zou Y, et al: Reduced efficiency of functional brain network underlying intellectual decline in patients with low-grade glioma. Neurosci Lett 543:27-31, 2013

105. Young MP: Objective analysis of the topological organization of the primate cortical visual system. Nature 358:152155,1992

106. Zalesky A, Fornito A, Bullmore E: On the use of correlation as a measure of network connectivity. Neuroimage 60:2096-2106, 2012

107. Zalesky A, Fornito A, Harding IH, Cocchi L, Yücel M, Pantelis C, et al: Whole-brain anatomical networks: does the choice of nodes matter? Neuroimage 50:970-983, 2010

108. Zeng LL, Shen H, Liu L, Wang L, Li B, Fang P, et al: Identifying major depression using whole-brain functional connectivity: a multivariate pattern analysis. Brain 135:1498-1507, 2012

\section{Disclosure}

The authors report no conflict of interest concerning the materials or methods used in this study or the findings specified in this paper.

\section{Author Contributions}

Conception and design: Hart, Suckling. Drafting the article: all authors. Critically revising the article: all authors. Reviewed submitted version of manuscript: all authors. Approved the final version of the manuscript on behalf of all authors: Hart. Figures and illustrations: Romero-Garcia.

\section{Correspondence}

Michael Hart, Division of Neurosurgery, Department of Clinical Neurosciences, Box 167, Cambridge Biomedical Campus, Cambridge CB2 0QQ, United Kingdom. email: mgh40@cam.ac.uk. 